\title{
Design and Analysis of a Novel Mechanism for the Morphing of Trailing Edge of an Aircraft Wing
}

\author{
Harun Levent Şahin ${ }^{1, *}$ and Yavuz Yaman ${ }^{2}$ \\ ${ }^{1}$ Graduate Research Assistant, Department of Aerospace Engineering, METU, Republic of Turkey \\ ${ }^{2}$ Professor, Department of Aerospace Engineering, METU, Republic of Turkey
}

\begin{abstract}
In this paper, design of a novel deployable scissor-structural mechanism (SSM) for active camber and chord morphing airfoil has been presented. The mechanism is created via combination of one four-bar linkage and various scissor-like elements (SLEs). The theory behind scissor-like elements and hierarchical design procedure are adequately explained. Following that design procedure, a scissor-structural mechanism is created in order to satisfy the desired airfoil shapes, which have different camber lines with minimum structural error. It is also possible to modify the chord length by changing the properties and types of the used SLEs. Modifying the design parameters of used SLEs, without increasing the degree-of-freedom (DOF) of the mechanism, will result in infinite number of results. With the help of error definition and developed computerroutine, the best scissor-structural mechanism which satisfy the required tasks properly can be detected.
\end{abstract}

\section{Introduction}

The nature teaches us that the birds accomplish various flight actions by altering their wings into various forms, so they benefit by getting more lift and thrust and reducing the drag [1]. Since the conventional aerial vehicles are generally optimized for a certain flight regime, the imitation of the nature can provides increasing efficiency in fuel consumption and aerodynamic noise of the morphing air vehicles[2, 3].

In commercial aviation, alternation of aircraft wings is achieved by conventional discrete flaps or slats [4]. Although these conventional systems are easy to apply, the presence of a sharp and discrete change in camber leads to a significant increase in drag over the wing, particularly at high lift coefficients, so with the increasing demand of efficiency; researchers focus on continuous deployment (without any clearance) of aircraft wing geometry, which often called as "morphing aircraft" [5]. It is proven that the camber of an airfoil has a significant impact on the aerodynamic forces generated under fluid flow [6]. Therefore, the most effective way to control the forces and moments that occur on aircraft wings is to change the camber of the airfoil [7]. For that reason, researchers in this

\footnotetext{
*Corresponding author: hlsahin@metu.edu.tr
} 
area generally focus and put studies on the topic of aircraft wing profile or simply "airfoil profile adjustment".

In the $21^{\text {st }}$ century, developments in material science, need of adaptive internal structures and lightweight actuation systems force researchers to study computational modeling and simulation of a novel reconfigurable aero-servo-elastic system [8]. From that point of view, one can conclude that airfoil profile adjustment is still a big problem waiting for an acceptable solution.

This paper presents a new methodology to design a deployable SSM for aircraft wings to camber/ decamber and resize the chord length. In the study it has been assumed that the aircraft wing skin and the internal structure provide the necessary strength and material properties.

\section{Theory}

Although they can be expanded to the three-dimension space with the help of special type of hinges, "scissor-structural mechanisms (SSM)" can be classified as the type of planar mechanisms; which are formed by a series of scissor-like elements (SLEs). If mechanisms have the characteristic of both of a mechanism (since they are movable, foldable, deployable) and of a structure (resist loads when they are fixed), they are called "structural mechanism" [9].

\subsection{Terms and definitions}

Scissor-like elements are called differently by various researchers. In this paper, Gantes' [10] terminology is used.

In the Fig. 1, the most basic form of a SLE is shown. In its basic form, two straight bars or links are connected to each other with a revolute joint. This location can be called as "pivot". In the same figure, end nodes, where a SLE can be assembled to another one, are shown as "hinge". The portion of a straight bar from pivot location to the end nodes are called as "section". Scissor-like elements are distinguished by imaginary lines which get through the hinge locations, which are called as "t-lines".

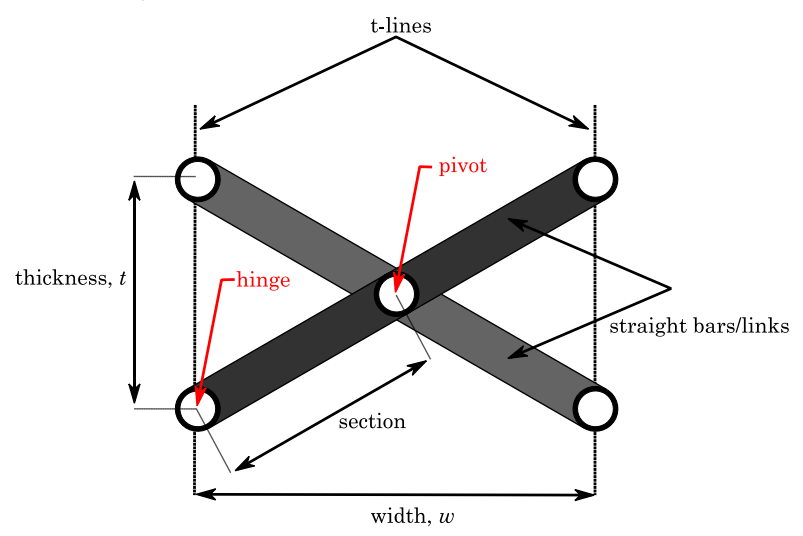

Fig. 1. A common scissor-like element.

Common property of SLEs is the inverse-proportion of the thickness and the width. It is clearly seen that, by changing the type of SLE, such inverse relation can take a complex form, which are used to stretch/shrink/ bend any geometry in any direction. This relation can be brought out by defining a "foldability vector" which connects midpoints between the left-hand-side (LHS) and right-hand-side (RHS) end nodes [11]. 


\subsection{Typology of scissor-like elements}

The typology of the scissor-like element determines the morphology and the movement characteristics of the structure. In general, there are four main categories of scissor-like elements which consist of only bar elements with revolute or prismatic joints: "translational scissor-like elements (t-SLE)", "polar scissor-like elements (p-SLE)", "angulated scissorlike elements (a-SLE)", and scissor-like elements which have additional degree-of-freedom a.k.a. "modified scissor-like elements (m-SLE)" [12]. In this paper, only t-SLEs and pSLEs are used to create proposed SSM.

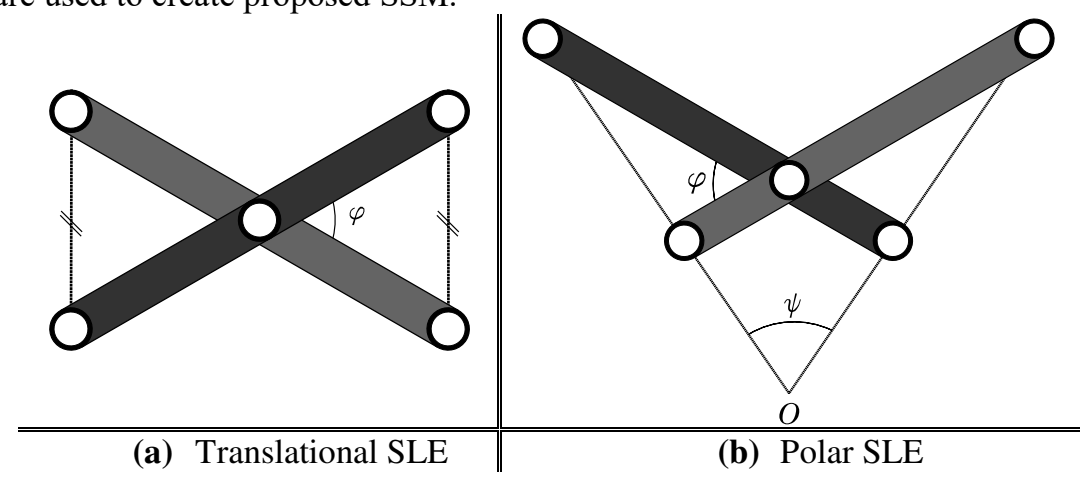

Fig. 2. Translational and polar scissor-like elements.

In the type of "translational SLEs", described t-lines remain parallel throughout the deployment. The most basic form of a translational SLE consists of two identical straight bars, pivoted to each other from their geometric centers and results in a linear motion in two-dimensional space. If straight bar lengths are changed then it is possible to generate curvilinear motion. In both case, it is also possible to obtain an irregular form by adding eccentricity to the pivot location.

When the imaginary t-lines do not remain parallel throughout the deployment process, then this type of elements are called "polar SLEs", a.k.a. curvilinear SLEs. Polar SLEs can also be classified in terms of straight-bar lengths and eccentricity.

\subsection{Kinematic analysis of scissor-structural mechanism}

In Fig. 3, a general planar SSM consisting of only translational and polar SLEs is shown. The "mobility" (the number of input parameters that must be independently controlled) a.k.a. "degree of freedom" (DOF) of the proposed SSM can be calculated by ChebychevGrübler-Kutzbach's formula, which is generally presented as follows [13]

$$
M=\lambda\left(n_{l}-n_{j}-1\right)+\sum_{i=1}^{n_{j}} f_{i}
$$

Assuming two of end nodes are grounded, if the mobility of the proposed SSM is calculated, one can get the result $M=0$, which means, the proposed SSM cannot move (behaves as a structure); therefore, in order to increase the DOF of the mechanism, one should set free one of the grounded joint or add an additional input linkage system. In this paper, an additional four-bar linkage is assumed to be attached from one of the grounded joint to manipulate the whole SSM which is represented by only output link of that four-bar linkage.

In order to find out the capability of the designed scissor-like structure and determine the inertial forces, kinematic analysis of the mechanism should be done properly. If a fourbar linkage is attached to manipulate the whole SSM, the system becomes a 1-DOF 
mechanism. In other words, one need to control only one parameter to manipulate the whole system. If the output angle of the four-bar linkage in terms of time is known; then, the position analysis of whole SSM can be done in terms of that output angle.

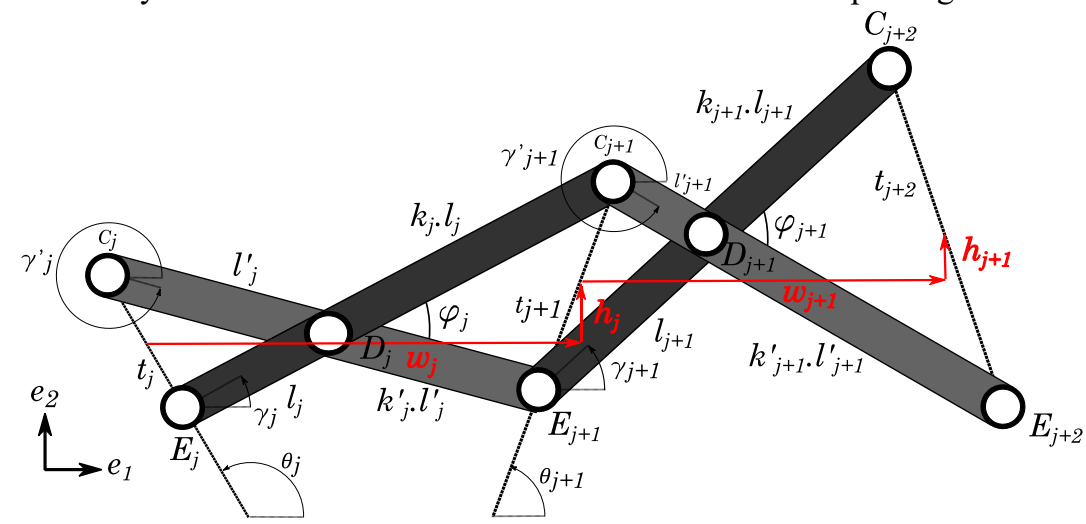

Fig. 3. A general planar SSM consisting of only translational and polar SLEs.

In Fig. $3, C_{j} E_{j+1}$ and $E_{j} C_{j+1}$ are two straight bars which form the $j^{\text {th }}$ SLE of the SSM. $l_{j}, k_{j} l_{j}$ and $l_{j}^{\prime}, k_{j}^{\prime} l_{j}^{\prime}$ represent section lengths, $\gamma_{j}$ and $\gamma_{j}^{\prime}$ represent orientations about global coordinates of those straight bars respectively. When another SLE is attached to the $j^{\text {th }}$ SLE, let $(j+1)^{t h}$ SLE, there occurs a closed loop $D_{j} C_{j+1} D_{j+1} E_{j+1}$, which is a "quadrilateral". The close-loop equation of that quadrilateral can be written in complex notation as

$$
k_{j} l_{j} e^{i \gamma_{j}}+l_{j+1}^{\prime} e^{i \gamma_{j+1}^{\prime}}=k_{j}^{\prime} l_{j}^{\prime} e^{i \gamma_{j}^{\prime}}+l_{j+1} e^{i \gamma_{j+1}}
$$

Equation (2) can be solved if orientation angles of $j^{\text {th }} \operatorname{SLE}\left(\gamma_{j}\right.$ and $\left.\gamma_{j}^{\prime}\right)$ are known. Assume that the angle $\gamma_{j}$ varies, then multiplying both sides of equation (2) with $e^{i \gamma_{j}^{\prime}}$ gives an equation in terms of $\varphi_{j}=\gamma_{j}-\gamma_{j}^{\prime}, \mu_{j+1} \equiv \gamma_{j+1}-\gamma_{j}^{\prime}$ and $\mu_{j+1}^{\prime} \equiv \gamma_{j+1}^{\prime}-\gamma_{j}^{\prime}$.

$$
k_{j} l_{j} e^{i \varphi_{j}}+l_{j+1}^{\prime} e^{i \mu_{j+1}^{\prime}}=k_{j}^{\prime} l_{j}^{\prime}+l_{j+1} e^{i \mu_{j+1}}
$$

In the equation (3), the angle $\mu^{\prime}{ }_{j+1}$ can also be eliminated by multiplying both sides of equation (3) with $e^{i \mu_{j+1}^{\prime}}$. Hence, the resulting equation takes the form of well-known Freudenstein equation when the Euler's identity of $e^{i \theta}=\cos (\theta)+i \sin (\theta)$ is applied.

$$
F\left(\varphi_{j}, \mu_{j+1}\right)=p_{1}+p_{2} \cos \left(\mu_{j+1}\right)-p_{3} \cos \left(\varphi_{j}\right)-\cos \left(\mu_{j+1}-\varphi_{j}\right)=0
$$

where Freudenstein parameters $\left(p_{1}, p_{2}, p_{3}\right)$ are

$$
p_{1} \equiv \frac{k_{j}^{\prime 2} l_{j}^{\prime 2}+k_{j}^{2} l_{j}^{2}-l_{j+1}^{\prime 2}+l_{j+1}^{2}}{2 k_{j} l_{j} l_{j+1}}, p_{2} \equiv \frac{k^{\prime}{ }_{j} l_{j}^{\prime}}{k_{j} l_{j}}, p_{3} \equiv \frac{k_{j}^{\prime} l_{j}^{\prime}}{l_{j+1}}
$$

Freudenstein equation (4) gives an implicit relation between the position variables $\varphi_{j}$ and $\mu_{j+1}$. This equation can be solved by applying half-tangent representation of the sine and cosine function of unknown $\mu_{j+1}$ and solving the arising quadratic equation in terms of $T \equiv \tan \left(\frac{\mu_{j+1}}{2}\right)$. 
From the designed four-bar linkage, the first orientation angles $\gamma_{1}, \gamma_{1}^{\prime}$, afterwards other orientation angles, $\left\{\gamma_{j}\right\}_{2}^{N},\left\{\gamma^{\prime}{ }_{j}\right\}_{2}^{N}$ can be determined.

\section{Design of a scissor-structural mechanism for the morphing of trailing edge of an aircraft wing}

Any planar SSM constructed by using proposed translational and polar SLEs, can generate three different 2D curves. As seen from the Fig. 4, those three curves are assumed to pass through joint locations $\left\{C_{j}\right\}_{1}^{N},\left\{D_{j}\right\}_{1}^{N},\left\{E_{j}\right\}_{1}^{N}$ respectively. This property of planar SSMs can be used to morph structures which are described by curves. In order to design a SSM to transform any planar structure which is characterized by two different curves to another shape, the total number of SLEs, their types and their parameters should be selected/optimized correctly to satisfy the both geometries. With developed computerroutine, several SSMs can be obtained and compared to find out the best SSM which has the minimum structural error which is defined as

$$
\operatorname{err}_{j}=\frac{d_{j}}{s}
$$

where $d_{j}$ is sum the shortest distances from the newest joint locations of $j^{\text {th }}$ SLE to the target curves, and $s$ is the characteristic dimension selected to reveal the percentage error.

Since all errors are positive, $\left(\left\{\operatorname{err}_{j}\right\}_{1}^{N} \geq 0\right)$, the mean error can be calculated as

$$
e r r_{m}=\frac{1}{N} \sum_{1}^{N} e r r_{j}
$$

which is the only parameter to compare different SSMs with each other.

In order to design a planar SSM for the morphing of trailing edge of an aircraft wing, the chord length of the airfoil can be taken as unity and characteristic dimension for convenience. Assuming the rear spar of the wing at the $60 \%$ of the chord length, starting from the rear spar to the trailing edge of the airfoil, various SSMs are designed with various parameters. In contrast to previous work [14], the links of proposed SSM are not remain parallel throughput the deployment.

The NACA 4412 is taken as the baseline airfoil, and it is aimed to morph this airfoil into NACA 8412. For three different total number of SLEs $(N=8,10,12)$, perturbing the design parameters, several SSMs have been designed. Those SSMs also investigated whether they satisfy the other airfoils (i.e. NACA 2412, NACA 6412) which have the same thickness and maximum camber location. Table 1 gives a summary of mean structural errors calculated:

Table 1. Mean structural errors for various SSMs with different number of SLEs.

\begin{tabular}{l||c|c|c} 
& $\boldsymbol{N}=\mathbf{8}$ & $\boldsymbol{N}=\mathbf{1 0}$ & $\boldsymbol{N}=\mathbf{1 2}$ \\
\hline \hline NACA 2412 & 0.0016 & 0.0015 & 0.0019 \\
\hline NACA 6412 & 0.0006 & 0.0008 & 0.0008 \\
\hline NACA 8412 & 0.0012 & 0.0017 & 0.0022
\end{tabular}


In the developed computer-routine, the user can define design parameters $N,\left\{w_{j}\right\}_{1}^{N}$, $\left\{\theta_{j}\right\}_{1}^{N},\left\{\psi_{j}\right\}_{1}^{N}$. Since the airfoil geometry encloses the SSM from upper and lower, the other design parameters $\left\{h_{j}\right\}_{1}^{N}$ and $\left\{t_{j}\right\}_{1}^{N}$ can automatically be adjusted. 
In Fig. 4, SSM with $N=8$ SLEs at its initial position is shown. In this case, mean t-line orientation angle is chosen a hundred degrees, $\bar{\theta}=100^{\circ}$; all SLEs are the type of p-SLE with constant $\left\{\psi_{j}\right\}_{1}^{N}=3^{\circ}$; the segmentation is done linearly (widths of each SLE is equal to each other).

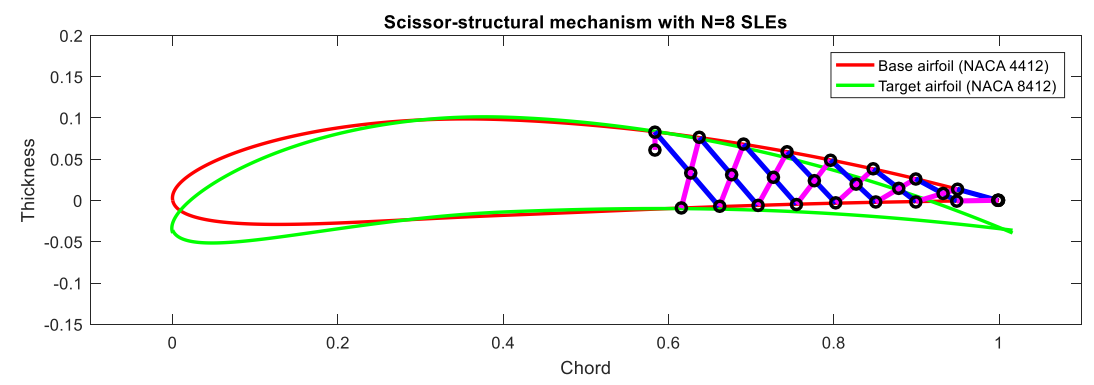

Fig. 4. Scissor-structural mechanism with $\boldsymbol{N}=\mathbf{8}$ SLEs at its initial position.

Fig. 5 shows that if the anchor link is rotated counter-clockwise, the mechanism adds decamber property to the aircraft wing. The same SSM with $N=8$ SLEs, can satisfy NACA 2412 and NACA 6412 profiles with $0.16 \%$ and $0.06 \%$ mean structural errors respectively.

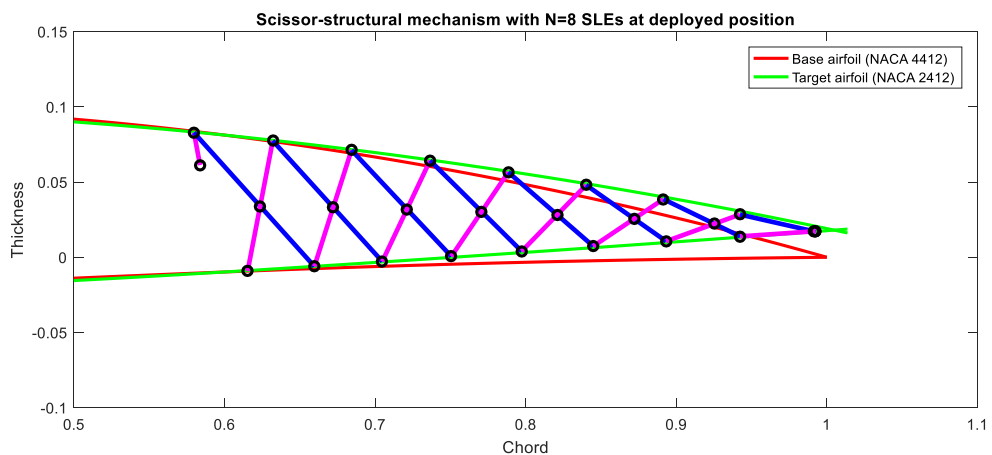

(a)

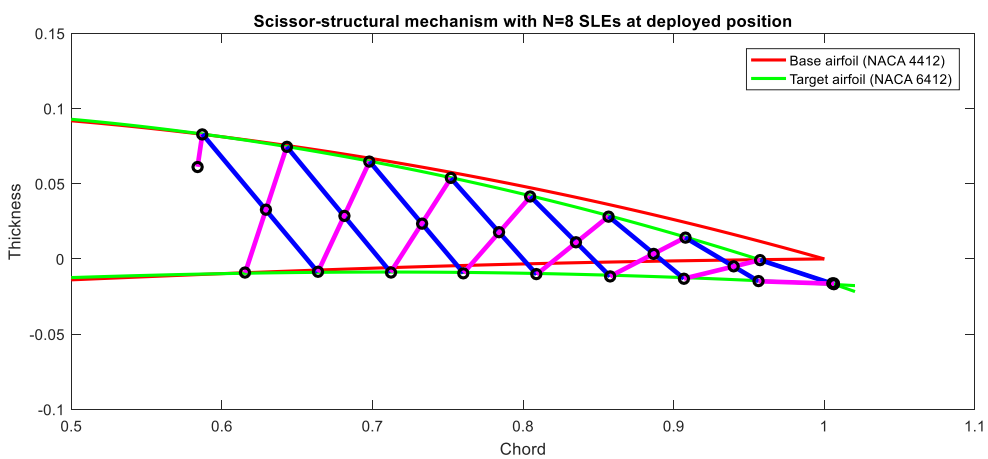

(b)

Fig. 5. Scissor-structural mechanism with $\boldsymbol{N}=\mathbf{8}$ SLEs at the deployed position when the target airfoil is (a) NACA 2412, (b) NACA 6412. 
As seen from Fig. 6a, when the anchor link is rotated $\phi=19^{\circ}$ clockwise, the designed SSM will satisfy the NACA 8412 geometry with $0.12 \%$ mean structural error. Fig. $6 \mathrm{~b}$ and Fig. $6 \mathrm{c}$ illustrate designed two different SSMs with $N=10$ and $N=12$ SLEs. In those cases, mean t-line is chosen perpendicular to the chord direction, $\bar{\theta}=90^{\circ}$, and the other parameters are hold the same. Then, when the anchor link is rotated $\phi=17^{\circ}$ and $\phi=27^{\circ}$ clockwise, the designed SSMs will satisfy the NACA 8412 geometry with $0.17 \%$ and $0.22 \%$ mean structural errors respectively.

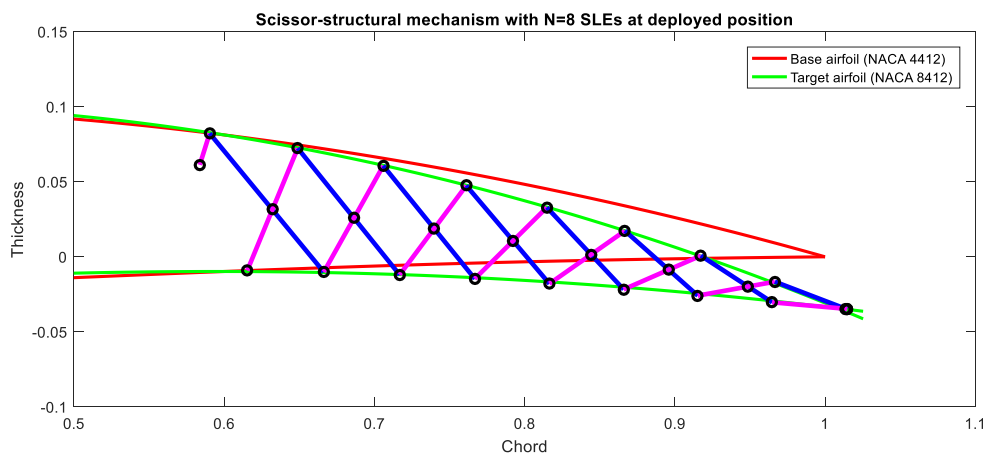

(a)

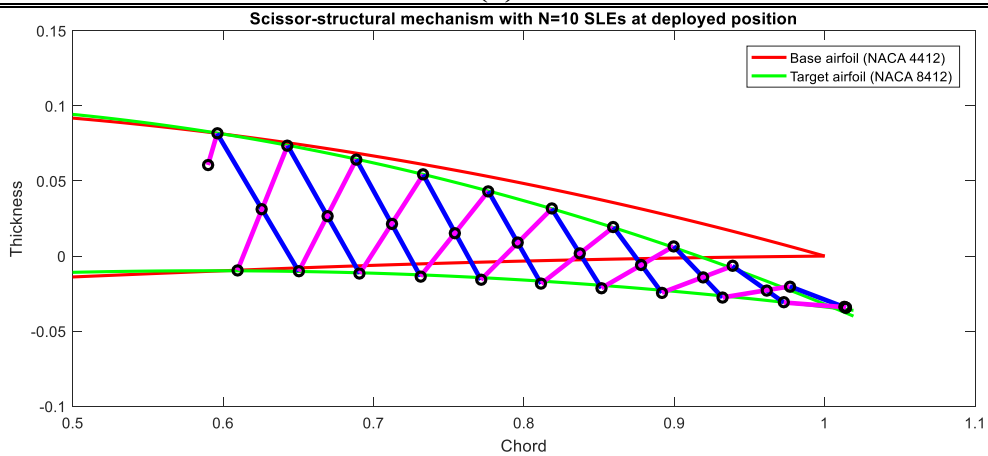

(b)

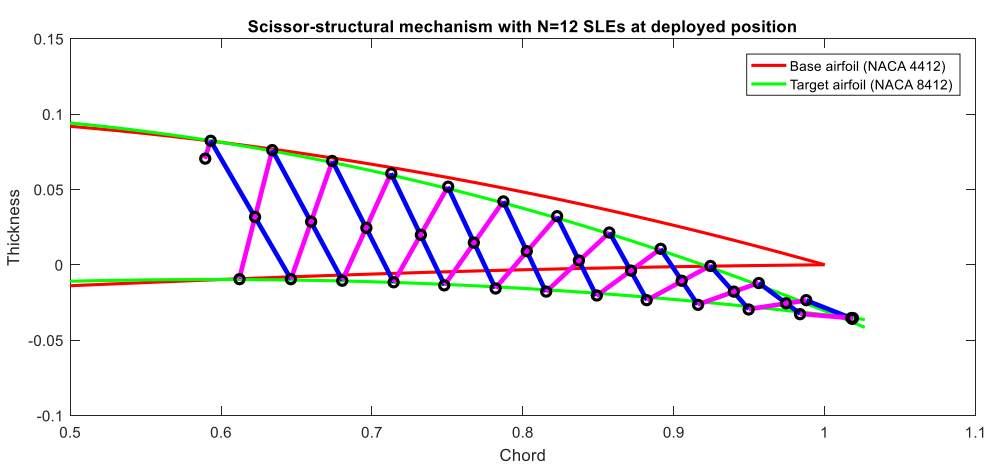

(c)

Fig. 6. Scissor-structural mechanisms with (a) $\boldsymbol{N}=\mathbf{8}$ SLEs, (b) $\boldsymbol{N}=\mathbf{1 0}$ SLEs, (c) $\boldsymbol{N}=\mathbf{1 2}$ SLEs at their deployed positions. 


\section{Conclusion}

This article presented an ongoing research about morphing of the trailing edge of an aircraft wing. Assuming an aircraft wing with a flexible skin which is able to follow the motion of the internal mechanism, a scissor-structural mechanism for the morphing of trailing edge of an aircraft wing is designed. In order to satisfy mobility requirements, a four-bar linkage mechanism is assumed to attached to the designed scissor-structural mechanism which is represented in the results by only anchor link.

As seen from the results, designed scissor-structural mechanisms for each case satisfy the baseline and target airfoil shapes successfully.

\section{References}

1 M. Ghommem, M.R. Hajj, P.S. Beran, I.K. Puri, Theor. Appl. Mech. 4 (2014) 1-7.

2 T. Weisshaar, Multifunct. Struct. / Integr. Sensors Antennas. (2006) O1-1 - O1-20.

3 M. Kintscher, M. Wiedemann, H.P. Monner, O. Heintze, T. Kühn, Int. J. Struct. Integr. 2 (2011) 383-405.

4 I. Chopra, AIAA J. 40 (2002) 2145-2187.

5 S. Barbarino, O. Bilgen, R.M. Ajaj, M.I. Friswell, D.J. Inman, J. Intell. Mater. Syst. Struct. 22 (2011) 823-877.

6 I.H. Abbott, A.E. Von Doenhoff, Press. 11 (1959) 693.

7 S. Murugan, B.K.S. Woods, M.I. Friswell, Aerosp. Sci. Technol. 42 (2015) 31-38.

8 A. De Gaspari, S. Ricci, J. Intell. Mater. Syst. Struct. 22 (2011) 1091-1111.

9 Y. Akgün, C.J. Gantes, W. Sobek, K. Korkmaz, K. Kalochairetis, Eng. Struct. 33 (2011) 1365-1376.

10 C.J. Gantes, J.J. Connor, R.D. Logcher, Y. Rosenfeld, Comput. Struct. 32 (1989) 661669.

11 T. Langbecker, Int. J. of Space Struct. 14 (1998) 1-15.

12 F. Maden, K. Korkmaz, Y. Akgün, Architectural Science Review 54 (2011) 246-257.

13 J. Zhao, Z. Feng, N. Ma, F. Chu, (Springer T, Springer-Verlag Berlin Heidelberg, 2014).

14 H.L. Şahin, Y. Yaman, in: 7th EASN Int. Conf. Innov. Eur. Aeronaut. Res. (Warsaw, 2017). 